\title{
A Numerical Technique for Solving Nonlinear Singularly Perturbed Delay Differential Equations
}

\author{
A.S.V. Ravi Kanth and P. Murali Mohan Kumar \\ Department of Mathematics, National Institute of Technology Kurukshetra \\ Kurukshetra, Haryana, India \\ E-mail(corresp.): asvravikanth@yahoo.com
}

Received April 28, 2017; revised December 5, 2017; accepted December 6, 2017

\begin{abstract}
This paper presents a numerical technique for solving nonlinear singularly perturbed delay differential equations. Quasilinearization technique is applied to convert the nonlinear singularly perturbed delay differential equation into a sequence of linear singularly perturbed delay differential equations. An exponentially fitted spline method is presented for solving sequence of linear singularly perturbed delay differential equations. Error estimates of the method is discussed. Numerical examples are solved to show the applicability and efficiency of the proposed scheme. Keywords: singularly perturbed problems, nonlinear delay problems, quasilinearization, parametric cubic spline.
\end{abstract}

AMS Subject Classification: 65L10; 65L11.

\section{Introduction}

In this paper, we consider the following nonlinear singularly perturbed delay differential equations is of the form:

$$
\varepsilon y^{\prime \prime}=g\left(x, y, y^{\prime}(x-\delta)\right) \quad \text { on }(0,1)
$$

under the interval and boundary conditions

$$
y(x)=\phi(x) \text { on }-\delta \leqslant x \leqslant 0, y(1)=\gamma,
$$

where $\varepsilon$ is a small singular perturbation parameter, $0<\varepsilon \ll 1$ and $\delta$ is the delay parameter of $o(\varepsilon)$. The solution $y(x)$ of the boundary value problem (1.1)-(1.2) must be continuous on $[0,1]$ and continuously differentiable on $(0,1)$. Suppose $g$ is smooth and satisfies the conditions,

This is an Open Access article distributed under the terms of the Creative Commons Attribution License (http://creativecommons.org/licenses/by/4.0/), which permits unrestricted use, distribution, and reproduction in any medium, provided the original author and source are credited. 
i. $\frac{\partial}{\partial y} g(x, y, z) \geqslant 0$ and $\frac{\partial}{\partial z} g(x, y, z) \leqslant 0$,

ii. $\left(\frac{\partial}{\partial y}-\frac{\partial}{\partial z}\right) g(x, y, z) \geqslant \alpha>0, \alpha$ is a positive constant,

iii. the growth condition $g(x, y, z)=O\left(|z|^{2}\right)$ as $z \rightarrow \infty$ for all $x \in[0,1]$ and all real $y$ and $z$.

For $\delta=0$, under the conditions listed above the equations (1.1)-(1.2) has a unique solution [10].

In general, the boundary value problems for singularly perturbed nonlinear delay differential equations play an essential role in explaining different applications like oceanic and atmosphere circulation [4], theory of non-premixed combustion [7], geodynamics [9], chemical reactions [18] etc.

In the past, the numerical study of linear singularly perturbed delay differential equations has been paid more attention $[5,6,14]$. But in recent years, there has been growing interest in solving nonlinear singularly perturbed delay differential equations. The solution of these problems can have steep exponential boundary layers. Classical approaches for solving such type of problems are inefficient due to the existence of boundary layer behavior when singular perturbation parameter $(\varepsilon)$ is goes to zero. It is significant to improve suitable numerical methods for these problems whose accuracy does not depend on $\varepsilon$. Lange and Miura [13] considered the boundary value problems for a singularly perturbed nonlinear differential equation with shift and discussed the existence and uniqueness of their solutions. Bartoszewski and Baranowska [2] have presented a fixed-point approach to solve boundary value problem for second order singularly perturbed delay differential equation. Kadalbajoo and Sharma [12] presented a finite difference method for solving the singularly perturbed nonlinear with delay differential equations. Kadalbajoo and Kumar [11] constructed B'Spline collocation method to solve singularly perturbed nonlinear system with delay differential equations. A numerical patching technique is presented for solving singularly perturbed nonlinear delay differential equations in [16].

A description of the contents of the paper is as follows. In Section 2, quasilinearization method and convergence analysis are discussed. Section 3 recalls the continuous problem. Derivation of the scheme is given in Section 4. Error estimates for the method is discussed in Section 5. Finally, the paper ends with computational results and discussion in Section 6.

\section{The quasilinearization method}

The quasilinearization technique [3], has been used to reduce the given nonlinear singularly perturbed delay differential equations (1.1)-(1.2) into a sequence of linear singularly perturbed delay differential equations. We choose a reasonable initial approximation for the function $y(x)$ in $g\left(x, y, y^{\prime}(x-\delta)\right)$, call it as 
$y^{(0)}(x)$ and expand $g\left(x, y, y^{\prime}(x-\delta)\right)$ around the function $y^{(0)}(x)$, we obtain

$$
\begin{aligned}
& g\left(x, y^{(1)}, y^{\prime(1)}(x-\delta)\right)=g\left(x, y^{(0)}, y^{\prime(0)}(x-\delta)\right) \\
& \quad+\left(y^{(1)}-y^{(0)}\right)\left(\frac{\partial g}{\partial y}\right)_{\left(x, y^{(0)}, y^{\prime(0)}\right)}+\left(y^{(1)}-y^{\prime(0)}\right)\left(\frac{\partial g}{\partial y^{\prime}}\right)_{\left(x, y^{(0)}, y^{\prime(0)}\right)}+\ldots
\end{aligned}
$$

In general we can write for $k=0,1, \ldots(k$ is iteration index $)$

$$
\begin{aligned}
& g\left(x, y^{(k+1)}, y^{(k+1)}(x-\delta)\right)=g\left(x, y^{(0)}, y^{(0)}(x-\delta)\right) \\
& +\left(y^{(k+1)}-y^{(k)}\right)\left(\frac{\partial g}{\partial y}\right)_{\left(x, y^{(0)}, y^{\prime(0)}\right)}+\left(y^{(k+1)}-y^{\prime(k)}\right)\left(\frac{\partial g}{\partial y^{\prime}}\right)_{\left(x, y^{(0)}, y^{\prime(0)}\right)}+\ldots
\end{aligned}
$$

Using the quasilinearization process, equations (1.1)-(1.2) becomes,

$$
\begin{gathered}
\varepsilon y^{\prime \prime(k+1)}(x)+\left(-\frac{\partial g^{(k)}}{\partial y^{\prime}}\right) y^{(k+1)}(x-\delta)+\left(-\frac{\partial g^{(k)}}{\partial y}\right) y^{(k+1)}(x) \\
=\left(g^{(k)}-y^{(k)} \frac{\partial g^{(k)}}{\partial y}-y^{\prime(k)}(x-\delta) \frac{\partial g^{(k)}}{\partial y^{\prime}}\right), \quad k=0,1, \ldots
\end{gathered}
$$

with

$$
y^{(k+1)}(x)=\phi(x), \quad-\delta \leqslant x \leqslant 0, \quad y^{(k+1)}(1)=\gamma
$$

and $g^{(k)}=g\left(x, y^{(k)}, y^{\prime(k)}(x-\delta)\right)$. Thus the equations (2.1)-(2.2) are linear in $y^{(k+1)}(x)$. Instead of solving the original problem (1.1)-(1.2), now we will solve a sequence of linear singularly perturbed delay differential equations given by (2.1)-(2.2) by using exponential fitting method which is introduced in Section 4. Theoretically, we require for a solution to the nonlinear problem,

$$
\lim _{k \rightarrow \infty} y^{(k)}(x)=y^{*}(x), \quad 0 \leqslant x \leqslant 1,
$$

where $y^{*}(x)$ is the solution of the nonlinear problem. Numerically, we require that

$$
\left|y^{(k+1)}(x)-y^{(k)}(x)\right|<\text { Tol. }, \quad 0 \leqslant x \leqslant 1 .
$$

Where Tol. is a small tolerance prescribed by us. We terminate the iteration once the tolerance test is reached and the profile $y^{(k+1)}(x)$ is the numerical solution of the nonlinear boundary value problem (1.1)-(1.2).

\subsection{Convergence analysis}

The convergence of the sequence $\left\langle y^{(k)}\right\rangle$ is obtained as follows. We denote $g\left(x, y, y^{\prime}(x-\delta)\right)$ as $g(y)$ throughout the convergence part for the sake of convenience. Consider the problem

$$
\begin{aligned}
& \varepsilon y^{\prime \prime}=g(y), \\
& y(x)=\phi(x), \quad-\delta \leqslant x \leqslant 0, \quad y(1)=\gamma .
\end{aligned}
$$


After quasilinearization technique, we get a sequence $\left\langle y^{(k)}\right\rangle$ of linear equations determined by the following recurrence relation

$$
\begin{gathered}
\varepsilon y^{\prime \prime(k+1)}(x)=g\left(y^{(k)}\right)+\left(y^{(k+1)}-y^{(k)}\right) g^{\prime}\left(y^{(k)}\right), \\
y^{(k+1)}(x)=\phi(x), \quad-\delta \leqslant x \leqslant 0, \quad y^{(k+1)}(1)=\gamma,
\end{gathered}
$$

where $g^{\prime}(y)=\frac{\partial g(y)}{\partial y}$. Let $y^{(0)}(x)$ be an initial approximation, then from equation (2.3), we have

$$
\varepsilon y^{\prime \prime(k)}(x)=g\left(y^{(k-1)}\right)+\left(y^{(k)}-y^{(k-1)}\right) g^{\prime}\left(y^{(k-1)}\right) .
$$

From equation (2.3) and equation (2.4), we have

$$
\begin{aligned}
\varepsilon\left(y^{(k+1)}-y^{(k)}\right)^{\prime \prime}(x)= & g\left(y^{(k)}\right)-g\left(y^{(k-1)}\right)-\left(y^{(k)}-y^{(k-1)}\right) g^{\prime}\left(y^{(k-1)}\right) \\
& +\left(y^{(k+1)}-y^{(k)}\right) g^{\prime}\left(y^{(k)}\right) .
\end{aligned}
$$

Equation (2.5) is a second order differential equation in $\left(y^{(k+1)}-y^{(k)}\right)$. Thus, the integral form of equation (2.5) by using Green's function is

$$
\begin{aligned}
& \varepsilon\left(y^{(k+1)}-y^{(k)}\right)(x)=\int_{0}^{1} G(x, s)\left[g\left(y^{(k)}\right)-g\left(y^{(k-1)}\right)\right. \\
& \left.-\left(y^{(k)}-y^{(k-1)}\right) g^{\prime}\left(y^{(k-1)}\right)+\left(y^{(k+1)}-y^{(k)}\right) g^{\prime}\left(y^{(k)}\right)\right] d s
\end{aligned}
$$

where $G(x, s)$ is the Green's function and determined by

$$
G(x, s)= \begin{cases}(x-1) s, & 0 \leqslant s \leqslant x \leqslant 1, \\ x(s-1), & 0 \leqslant x \leqslant s \leqslant 1,\end{cases}
$$

where $\max _{x, s}|G(x, s)|=1 / 4$. By the mean value theorem, we have

$$
g\left(y^{(k)}\right)-g\left(y^{(k-1)}\right)=\left(y^{(k)}-y^{(k-1)}\right) g^{\prime}\left(y^{(k-1)}\right)+\frac{\left(y^{(k)}-y^{(k-1)}\right)^{2}}{2} g^{\prime \prime}(\theta),
$$

where $y^{(k-1)} \leqslant \theta \leqslant y^{(k)}$. Substituting equation (2.7) into equation (2.6), we obtain

$$
\begin{aligned}
\varepsilon\left(y^{(k+1)}-y^{(k)}\right)= & \int_{0}^{1} G(x, s)\left[\left(y^{(k)}-y^{(k-1)}\right)^{2} g^{\prime \prime}(\theta) / 2\right. \\
& \left.+\left(y^{(k+1)}-y^{(k)}\right) g^{\prime}\left(y^{(k)}\right)\right] d s .
\end{aligned}
$$

Let

$$
\max _{\|y\| \leqslant 1} g^{\prime}(y)=a_{1}, \max _{\|y\| \leqslant 1} g^{\prime \prime}(y)=a_{2} .
$$

Taking the maximum of the moduli over the domain of consideration on both sides of equation (2.8), we get

$$
\begin{aligned}
\max _{0 \leqslant x \leqslant 1}\left|\left(y^{(k+1)}-y^{(k)}\right)(x)\right| & \leqslant \frac{1}{4 \varepsilon} \int_{0}^{1}\left[\max _{0 \leqslant x \leqslant 1} \frac{\left(y^{(k)}-y^{(k-1)}\right)^{2}}{2} \max _{0 \leqslant x \leqslant 1}\left|g^{\prime \prime}(\theta)\right|\right. \\
& \left.+\max _{0 \leqslant x \leqslant 1}\left|y^{(k+1)}-y^{(k)}\right| \max _{0 \leqslant x \leqslant 1}\left|g^{\prime}\left(y^{(k)}\right)\right|\right] d s .
\end{aligned}
$$


A simplification yields,

$$
\max _{0 \leqslant x \leqslant 1}\left|\left(y^{(k+1)}-y^{(k)}\right)(x)\right| \leqslant K_{1} \max _{0 \leqslant x \leqslant 1}\left(y^{(k)}-y^{(k-1)}\right)^{2},
$$

where

$$
K_{1}=a_{2} /\left(8 \varepsilon\left(1-a_{1} / 4 \varepsilon\right)\right)<1 .
$$

This proves that the sequence $\left\langle y^{(k)}\right\rangle$ of linear equations converges quadratically provided $K_{1}<1$. Therefore, to obtain the approximate solution of the nonlinear singularly perturbed delay differential equations (1.1) and (1.2), it is sufficient to approximate the solution of the sequence of linear singularly perturbed delay differential equation of the form

$$
\varepsilon y^{\prime \prime(k+1)}(x)+a^{(k)}(x) y^{(k+1)}(x-\delta)+b^{(k)}(x) y^{(k+1)}(x)=c^{(k)}(x),
$$

$k=0,1, \ldots$ with

$$
y^{(k+1)}(x)=\phi(x), \quad-\delta \leqslant x \leqslant 0, \quad y^{(k+1)}(1)=\gamma,
$$

where

$$
\begin{aligned}
& a^{(k)}(x)=-\frac{\partial g^{(k)}}{\partial y^{\prime}}, \quad b^{(k)}(x)=-\frac{\partial g^{(k)}}{\partial y} \\
& c^{(k)}(x)=g^{(k)}-y^{(k)} \frac{\partial g^{(k)}}{\partial y}-y^{(k)}(x-\delta) \frac{\partial g^{(k)}}{\partial y^{\prime}} .
\end{aligned}
$$

\section{Continuous problem}

When the delay parameter is of small order of the singular perturbation parameter i.e., $(\delta<\varepsilon)$, an application of Taylor's series expansion for the term $y^{\prime(k+1)}(x-\delta)$ in equation (2.9) yields,

$$
L_{\pi} \equiv \varepsilon y^{\prime \prime(k+1)}(x)+p^{(k)}(x) y^{(k+1)}(x)+q^{(k)}(x) y^{(k+1)}(x)=r^{(k)}(x),
$$

$k=0,1, \ldots$, where

$$
\begin{aligned}
& p^{(k)}(x)=\frac{a^{(k)}(x)}{1-\frac{\delta}{\varepsilon} a^{(k)}(x)}, \quad q^{(k)}(x)=\frac{b^{(k)}(x)}{1-\frac{\delta}{\varepsilon} a^{(k)}(x)}, \\
& r^{(k)}(x)=\frac{g^{(k)}-y^{(k)} \frac{\partial g^{(k)}}{\partial y}-\left[y^{\prime(k)}(x)-\delta y^{\prime \prime(k)}(x)\right] \frac{\partial g^{(k)}}{\partial y^{\prime}}}{1-\frac{\delta}{\varepsilon} a^{(k)}(x)}
\end{aligned}
$$

with

$$
y^{(k+1)}(0)=\phi(0)=\phi_{0}, \quad y^{(k+1)}(1)=\gamma .
$$

When $\delta$ is zero i.e., $(\delta=0)$, the solution of the above problem exhibits boundary layer on the left or right side of the interval depending on the sign of the coefficient $p^{(k)}(x)$, i.e., according as $p^{(k)}(x)>0$ or $p^{(k)}(x)<0$ respectively. Here we assume that $p^{(k)}(x) \geqslant p^{*}>0$ and $q^{(k)}(x) \leqslant-q^{*}<0$ for some positive constants $p^{*}, q^{*}$.

The operator $L_{\pi}=\varepsilon \frac{\mathrm{d}^{2}}{\mathrm{~d} x^{2}}+p^{(k)}(x) \frac{\mathrm{d}}{\mathrm{d} x}+q^{(k)}(x) I$ in (3.1) satisfies the following Lemma 1. 
Lemma 1. Suppose $\varphi(x)$ is a smooth function satisfying $\varphi^{(k+1)}(0) \geqslant 0$, $\varphi^{(k+1)}(1) \geqslant 0$. Then $L_{\pi} \varphi^{(k+1)}(x) \leqslant 0, \forall x \in(0,1)$ implies $\varphi^{(k+1)}(x) \geqslant 0$, $\forall x \in[0,1], k=0,1, \ldots$

Proof. Let $m \in[0,1]$ be such that $\varphi^{(k+1)}(m)<0$ and

$$
\varphi^{(k+1)}(m)=\min _{x \in[0,1]} \varphi^{(k+1)}(x) .
$$

Clearly $m \notin\{0,1\}$, therefore $\varphi^{\prime(k+1)}(m)=0$ and $\varphi^{\prime \prime(k+1)}(m) \geqslant 0$.

Now consider,

$\left.L_{\pi} \varphi^{(k+1)}(x)\right|_{x=m}=\varepsilon \varphi^{\prime \prime(k+1)}(m)+p^{(k)}(m) \varphi^{\prime(k+1)}(m)+q^{(k)}(m) \varphi^{(k+1)}(m)>0$,

which is contradictory to our assumption. Thus $\varphi^{(k+1)}(x) \geqslant 0, \forall x \in[0,1]$.

Lemma 2. Let $y^{(k+1)}(x)$ be the solution of the problem (3.1) and (3.2) then we have

$$
\left\|y^{(k+1)}\right\| \leqslant\left(q^{*}\right)^{-1}\left\|r^{(k)}\right\|+\max \left(\left|\phi_{0}\right|,|\gamma|\right), \quad k=0,1, \ldots,
$$

where $\|\cdot\|$ is the $L_{\infty}$ norm given by $\left\|y^{(k+1)}\right\|=\max _{0 \leqslant x \leqslant 1}\left|y^{(k+1)}(x)\right|$.

Proof. Let $\varphi^{ \pm(k+1)}(x)$ be two barrier functions defined by

$$
\varphi^{ \pm(k+1)}(x)=\left(q^{*}\right)^{-1}\|r\|+\max \left(\left|\varphi_{0}\right|,|\gamma|\right) \pm y^{(k+1)}(x) .
$$

Then this implies

$$
\begin{aligned}
\varphi^{ \pm(k+1)}(0) & =\left(q^{*}\right)^{-1}\left\|r^{(k)}\right\|+\max \left(\left|\phi_{0}\right|,|\gamma|\right) \pm y^{(k+1)}(0) \\
& =\left(q^{*}\right)^{-1}\left\|r^{(k)}\right\|+\max \left(\left|\phi_{0}\right|,|\gamma|\right) \pm \phi_{0} \geqslant 0 \\
\varphi^{ \pm(k+1)}(1) & =\left(q^{*}\right)^{-1}\left\|r^{(k)}\right\|+\max \left(\left|\phi_{0}\right|,|\gamma|\right) \pm y^{(k+1)}(1) \\
& =\left(q^{*}\right)^{-1}\left\|r^{(k)}\right\|+\max \left(\left|\phi_{0}\right|,|\gamma|\right) \pm \gamma \geqslant 0
\end{aligned}
$$

and

$$
\begin{aligned}
L_{\pi} \varphi^{ \pm(k+1)}(x) & =\varepsilon\left(\varphi^{ \pm(k+1)}(x)\right)^{\prime \prime}+p^{(k)}(x)\left(\varphi^{ \pm(k+1)}(x)\right)^{\prime}+q^{(k)}(x) \varphi^{ \pm(k+1)}(x) \\
& =q^{(k)}(x)\left[\left(q^{*}\right)^{-1}\left\|r^{(k)}\right\|+\max \left(\left|\phi_{0}\right|,|\gamma|\right)\right] \pm L_{\pi} y^{(k+1)}(x) \\
& =q^{(k)}(x)\left[\left(q^{*}\right)^{-1}\left\|r^{(k)}\right\|+\max \left(\left|\phi_{0}\right|,|\gamma|\right)\right] \pm r^{(k)}(x) .
\end{aligned}
$$

As $q^{(k)}(x) \leqslant-q^{*}<0$ implies $q^{(k)}(x)\left(q^{*}\right)^{-1} \leqslant-1$ and since $\left\|r^{(k)}\right\| \geqslant r^{(k)}(x)$, we have $L_{\tau} \varphi^{(k+1)} \leqslant 0, \forall x \in[0,1]$. By using Lemma 1 , we obtain the required estimate. 
Lemma 3. If $y^{(k+1)}$ satisfies equations $(3.1)-(3.2)$, then

$$
y^{(k+1)}(x)=u^{(k+1)}(x)+v^{(k+1)}(x)+w^{(k+1)}(x), k=0,1, \ldots,
$$

where

$$
\begin{aligned}
u^{(k+1)}(x) & =-\frac{\varepsilon y^{\prime(k+1)}(0)}{p^{(k)}(0)} \exp \left(\frac{-p^{(k)}(0) x}{\varepsilon}\right) \\
v^{(k+1)}(x) & =-\frac{\varepsilon y^{\prime(k+1)}(1)}{p^{(k)}(1)} \exp \left(\frac{-p^{(k)}(1)(1-x)}{\varepsilon}\right), \\
\left|w^{(s)(k+1)}(x)\right| & \leqslant M\left\{1+\varepsilon^{-s+1} \exp \left[\frac{-c_{1}(1-x)}{\varepsilon}\right]\right\}, s=0(1) 4,
\end{aligned}
$$

where $c_{1}$ is constant, $M$ is a positive constant independent of $h$ and $\varepsilon$.

From the theory of singular perturbations [15, pp. 22-26], it is known that the solution of (3.1) with (3.2) is of the form

$y^{(k+1)}(x) \approx y_{0}^{(k+1)}(x)+\frac{p^{(k)}(0)}{p^{(k)}(x)}\left(\phi_{0}-y_{0}^{(k+1)}(0)\right) \exp \left(-\int_{0}^{x} \frac{p^{(k)}(s)}{\varepsilon} d s\right)+O(\varepsilon)$,

where $y_{0}^{(k+1)}(x)$ is the solution of reduced problem

$$
p^{(k)}(x) y_{0}^{(k+1)}(x)+q^{(k)}(x) y_{0}^{(k+1)}(x)=r^{(k)}(x) \text { with } y_{0}^{(k+1)}(1)=\gamma .
$$

We first divide the interval $[0,1]$ into $N$ equal subintervals, each of length $h$. Let $0=x_{0}<x_{1}<\ldots<x_{N}=1$ be the points such that $x_{i}=i h, i=0,1, \cdots, N$. From (3.4) as $h \rightarrow 0$, we obtain

$$
\lim _{h \rightarrow 0} y^{(k+1)}(i h) \approx y_{0}^{(k+1)}(i h)+\left(\phi_{0}-y_{0}^{(k+1)}(0)\right) \exp \left(-p^{(k)}(0) i \rho\right)+O(\varepsilon),
$$

where $\rho=h / \varepsilon$. Now introducing an exponentially fitting factor $\sigma$ into equation (3.1), we get

$$
\sigma \varepsilon y^{\prime \prime(k+1)}(x)+p^{(k)}(x) y^{\prime(k+1)}(x)+q^{(k)}(x) y^{(k+1)}(x)=r^{(k)}(x), k=0,1, \ldots
$$

with

$$
y^{(k+1)}(0)=\phi_{0}, \quad y^{(k+1)}(1)=\gamma
$$

The fitting factor $\sigma$ is to be determined in such a way that the solution of equations (3.6)-(3.7) converges uniformly to the solution of equations (2.9)(2.10).

\section{Derivation of the scheme}

Let $x_{0}=a, x_{i}=a+i h, x_{N}=b, h=(b-a) / N$. A function $S^{(k+1)}(x, \tau)$ of class $C^{2}[a, b]$ which interpolates $y^{(k+1)}(x)$ at the mesh point $x_{i}$ depends on a parameter $\tau$, reduces to cubic spline in $[a, b]$ as $\tau \rightarrow 0$ is termed as parametric 
cubic spline function. The spline function $S^{(k+1)}(x, \tau)=S^{(k+1)}(x)$ satisfying in $\left[x_{i}, x_{i+1}\right]$, the differential equation

$$
\begin{aligned}
& S^{\prime \prime(k+1)}(x)+\tau S^{(k+1)}(x)=\left[S^{\prime \prime(k+1)}\left(x_{i}\right)+\tau S^{(k+1)}\left(x_{i}\right)\right] \frac{\left(x_{i+1}-x\right)}{h} \\
& +\left[S^{\prime \prime(k+1)}\left(x_{i+1}\right)+\tau S^{(k+1)}\left(x_{i+1}\right)\right] \frac{\left(x-x_{i}\right)}{h}
\end{aligned}
$$

where $S^{(k+1)}\left(x_{i}\right)=y_{i}^{(k+1)}$ and $\tau>0$ is termed as cubic spline in compression. Following Aziz and Khan [1], we obtain the tridiagonal system

$$
h^{2}\left(\lambda_{1} M_{i-1}^{(k+1)}+2 \lambda_{2} M_{i}^{(k+1)}+\lambda_{1} M_{i+1}^{(k+1)}\right)=y_{i-1}^{(k+1)}-2 y_{i}^{(k+1)}+y_{i+1}^{(k+1)},
$$

where $i=1, \ldots, N-1$,

$$
\begin{aligned}
& \lambda_{1}=\frac{1}{\lambda^{2}}\left(1-\frac{\lambda}{\sin \lambda}\right), \quad \lambda_{2}=\frac{1}{\lambda^{2}}(\lambda \cot \lambda-1), \\
& \lambda=h \tau^{1 / 2}, \quad M_{j}^{(k+1)}=y^{\prime \prime(k+1)}\left(x_{j}\right), \quad j=i, i \pm 1, \quad k=0,1, \ldots
\end{aligned}
$$

The consistency relation for (4.1) leads to the equation $\lambda_{1}+\lambda_{2}=\frac{1}{2}$, which may also be expressed as $\frac{\lambda}{2}=\tan \frac{\lambda}{2}$. This equation has a zero root and an infinite number of non-zero roots, the small positive being $\lambda=8.986818916 \ldots$ Substituting

$$
\sigma \varepsilon M_{j}^{(k+1)}=r^{(k)}\left(x_{j}\right)-p^{(k)}\left(x_{j}\right) y^{(k+1)}\left(x_{j}\right)-q^{(k)}\left(x_{j}\right) y^{(k+1)}\left(x_{j}\right), \quad j=i, i \pm 1
$$

into equation (4.1), and using the following approximations for first derivative of $y_{i}^{(k+1)}$

$$
\begin{aligned}
y_{i}^{\prime(k+1)} & \approx \frac{y_{i+1}^{(k+1)}-y_{i-1}^{(k+1)}}{2 h}, \quad y_{i+1}^{\prime(k+1)} \approx \frac{3 y_{i+1}^{(k+1)}-4 y_{i}^{(k+1)}+y_{i-1}^{(k+1)}}{2 h}, \\
y_{i-1}^{\prime(k+1)} & \approx \frac{-y_{i+1}^{(k+1)}+4 y_{i}^{(k+1)}-3 y_{i-1}^{(k+1)}}{2 h}
\end{aligned}
$$

we get the following difference scheme

$$
E_{i}^{(k)} y_{i-1}^{(k+1)}-F_{i}^{(k)} y_{i}^{(k+1)}+G_{i}^{(k)} y_{i+1}^{(k+1)}=H_{i}^{(k)}, \quad i=1, \ldots, N-1, \quad k \geq 0,
$$

where

$$
\begin{aligned}
E_{i}^{(k)} & =\frac{\sigma}{\rho}-\frac{3 \lambda_{1}}{2} p_{i-1}^{(k)}-\lambda_{2} p_{i}^{(k)}+\frac{\lambda_{1}}{2} p_{i+1}^{(k)}+\lambda_{1} h q_{i-1}^{(k)}, \\
F_{i}^{(k)} & =\frac{2 \sigma}{\rho}-2 \lambda_{1} p_{i-1}^{(k)}+2 \lambda_{1} p_{i+1}^{(k)}-2 \lambda_{2} h q_{i}^{(k)}, \\
G_{i}^{(k)} & =\frac{\sigma}{\rho}-\frac{\lambda_{1}}{2} p_{i-1}^{(k)}+\lambda_{2} p_{i}^{(k)}+\frac{3 \lambda_{1}}{2} p_{i+1}^{(k)}+\lambda_{1} h q_{i+1}^{(k)}, \\
H_{i}^{(k)} & =\lambda_{1} h r_{i-1}^{(k)}+2 \lambda_{2} h r_{i}^{(k)}+\lambda_{1} h r_{i+1}^{(k)} .
\end{aligned}
$$

Equation (4.2) forms a set of tridiagonal system of $(N-1)$ equations with $(N+$ 1) unknowns. These $(N-1)$ set of equations together with the equation (3.7) are sufficient to solve the system by using Thomas algorithm. 


\subsection{Determination of fitting factor}

Taking the limit as $h \rightarrow 0$ in equation (4.2), we obtain

$$
\begin{aligned}
& \lim _{h \rightarrow 0}\left(\frac{\sigma}{\rho}\left(y^{(k+1)}(i h+h)-2 y^{(k+1)}(i h)+y^{(k+1)}(i h-h)\right)\right) \\
& +\left(\lambda_{1}+\lambda_{2}\right) \lim _{h \rightarrow 0}\left(p^{(k)}(i h)\left(y^{(k+1)}(i h+h)-y^{(k+1)}(i h-h)\right)\right)=0 .
\end{aligned}
$$

We used the fact that $r^{(k)}\left(x_{i}\right)-q^{(k)}\left(x_{i}\right) y^{(k+1)}\left(x_{i}\right)$ is bounded. Substituting equation (3.5) into equation (4.3) and then simplifying, we get

$$
\sigma=p^{(k)}(0) \rho\left(\lambda_{1}+\lambda_{2}\right) \operatorname{coth}\left(\frac{p^{(k)}(0) \rho}{2}\right), \quad k=0,1, \ldots
$$

\section{$5 \quad$ Error estimates}

In this section, we derive the truncation error for the proposed scheme. The truncation error of the numerical scheme is given by

$$
\begin{gathered}
\tau_{i}\left(y^{(k)}\right)=\left[E_{i}^{(k)} y^{(k+1)}\left(x_{i-1}\right)-F_{i}^{(k)} y^{(k+1)}\left(x_{i}\right)+G_{i}^{(k)} y^{(k+1)}\left(x_{i+1}\right)\right] \\
-\left[\lambda_{1} h r^{(k)}\left(x_{i-1}\right)+2 \lambda_{2} h r^{(k)}\left(x_{i}\right)+\lambda_{1} h r^{(k)}\left(x_{i+1}\right)\right] \\
i=1(1) N-1, k=0,1, \ldots
\end{gathered}
$$

Using the equation (3.6) for $r^{(k)}(x)$ in the above equation (5.1), we obtain

$$
\begin{gathered}
\tau_{i}\left(y^{(k)}\right)=\left[E_{i}^{(k)} y^{(k+1)}\left(x_{i-1}\right)-F_{i}^{(k)} y^{(k+1)}\left(x_{i}\right)+G_{i}^{(k)} y^{(k+1)}\left(x_{i+1}\right)\right] \\
-\lambda_{1} h\left[\left(\sigma \varepsilon y^{\prime \prime(k+1)}\left(x_{i-1}\right)+p_{i-1}^{(k)} y^{\prime(k+1)}\left(x_{i-1}\right)+q_{i-1}^{(k)} y^{(k+1)}\left(x_{i-1}\right)\right)\right] \\
-2 \lambda_{2} h\left[\left(\sigma \varepsilon y^{\prime \prime(k+1)}\left(x_{i}\right)+p_{i}^{(k)} y^{\prime(k+1)}\left(x_{i}\right)+q_{i}^{(k)} y^{(k+1)}\left(x_{i}\right)\right)\right] \\
-\lambda_{1} h\left[\left(\sigma \varepsilon y^{\prime \prime(k+1)}\left(x_{i+1}\right)+p_{i+1}^{(k)} y^{\prime(k+1)}\left(x_{i+1}\right)+q_{i+1}^{(k)} y^{(k+1)}\left(x_{i+1}\right)\right)\right] .
\end{gathered}
$$

An application of Taylor series expansion for $y^{\prime \prime(k+1)}\left(x_{i-1}\right), y^{\prime \prime(k+1)}\left(x_{i+1}\right)$, $y^{\prime(k+1)}\left(x_{i-1}\right), y^{\prime(k+1)}\left(x_{i+1}\right), y^{(k+1)}\left(x_{i-1}\right)$ and $y^{(k+1)}\left(x_{i+1}\right)$ in the above expression (5.2), we have for $k \geq 0$ :

$$
\tau_{i}\left(y^{(k+1)}\right)=T_{0} y_{i}^{(k+1)}+T_{1} y_{i}^{\prime(k+1)}+T_{2} y_{i}^{\prime \prime(k+1)}+T_{3} y_{i}^{\prime \prime \prime(k+1)}+R e m .,
$$

where

$$
\begin{aligned}
T_{0}= & \left(E_{i}^{(k)}-F_{i}^{(k)}+G_{i}^{(k)}\right)-\left(\lambda_{1} h q_{i-1}^{(k)}+2 \lambda_{2} h q_{i}^{(k)}+\lambda_{1} h q_{i+1}^{(k)}\right), \\
T_{1}= & h\left(-E_{i}^{(k)}+G_{i}^{(k)}\right)-\left(\lambda_{1} h\left(p_{i-1}^{(k)}+p_{i+1}^{(k)}\right)+2 \lambda_{2} h p_{i}^{(k)}\right. \\
& \left.-\lambda_{1} h^{2}\left(q_{i-1}^{(k)}-q_{i+1}^{(k)}\right)\right),
\end{aligned}
$$




$$
\begin{aligned}
T_{2}= & \frac{h^{2}}{2 !}\left(E_{i}^{(k)}+G_{i}^{(k)}\right)-\left(2\left(\lambda_{1}+\lambda_{2}\right) h \sigma \varepsilon-\lambda_{1} h^{2}\left(p_{i-1}^{(k)}-p_{i+1}^{(k)}\right)\right. \\
& \left.+\lambda_{1} \frac{h^{3}}{2 !}\left(q_{i-1}^{(k)}+q_{i+1}^{(k)}\right)\right)=\frac{h \sigma \varepsilon}{2}\left(\frac{1}{2}-\left(\lambda_{1}+\lambda_{2}\right)\right), \\
T_{3}= & \frac{h^{3}}{3 !}\left(-E_{i}^{(k)}+G_{i}^{(k)}\right)-\left(\lambda_{1} \frac{h^{3}}{2 !}\left(p_{i-1}^{(k)}+p_{i+1}^{(k)}\right)-\lambda_{1} \frac{h^{4}}{3 !}\left(q_{i-1}^{(k)}-q_{i+1}^{(k)}\right)\right), \\
= & \frac{h^{3}}{3}\left(\lambda_{1} p_{i-1}^{(k)}-\lambda_{2} p_{i}^{(k)}+\lambda_{1} p_{i+1}^{(k)}\right) .
\end{aligned}
$$

It can be easily seen that,

$$
T_{0}=T_{1}=T_{2}=0, \quad\left|T_{3}\right| \leqslant M h^{3}, \quad \text { when } \quad \lambda_{1}+\lambda_{2}=\frac{1}{2} .
$$

Now, from Lemma 3, we have

$$
u_{j}^{\prime \prime \prime(k+1)}=-\left(\frac{p^{(k)}(0)}{\varepsilon}\right)^{2} y^{\prime}(0) \exp \left(\frac{-p^{(k)}(0) x_{j}}{\varepsilon}\right),
$$

therefore,

$$
\left|\tau_{j}\left(u^{(k+1)}\right)\right| \leqslant \frac{M h^{3}}{\varepsilon^{2}} \exp \left(\frac{-p^{(k)}(0) x_{j}}{\varepsilon}\right) .
$$

Similarly,

$$
\left|\tau_{j}\left(v^{(k+1)}\right)\right| \leqslant \frac{M h^{3}}{\varepsilon^{2} a} \exp \left(\frac{-p^{(k)}(1)\left(1-x_{j}\right)}{\varepsilon}\right)
$$

and

$$
\begin{aligned}
& \left|w_{j}^{(3)(k+1)}(x)\right| \leqslant M\left\{1+\varepsilon^{-2} \exp \left(\frac{-c_{1}\left(1-x_{j}\right)}{\varepsilon}\right)\right\}, \\
& \left|\tau_{j}\left(w^{(k+1)}\right)\right| \leqslant M h^{3}\left\{1+\varepsilon^{-2} \exp \left(\frac{-c_{1}\left(1-x_{j}\right)}{\varepsilon}\right)\right\} .
\end{aligned}
$$

Now,

$$
\begin{aligned}
& \tau_{j}\left(y^{(k+1)}\right)=\tau_{j}\left(u^{(k+1)}\right)+\tau_{j}\left(v^{(k+1)}\right)+\tau_{j}\left(w^{(k+1)}\right) \\
& \left|\tau_{j}\left(y^{(k+1)}\right)\right| \leqslant \frac{M h^{3}}{\varepsilon^{2}}\left[1+\exp \left(\frac{-p^{(k)}(0) x_{j}}{\varepsilon}\right)+\exp \left(\frac{-p^{(k)}(1)\left(1-x_{j}\right)}{\varepsilon}\right)\right] .
\end{aligned}
$$

Equation (4.2) can be written in the matrix form

$$
A Y^{(k+1)}=B, \quad k=0,1, \ldots,
$$

where $A$ is the matrix of the system (4.2), $Y^{(k+1)}$ and $B$ are the corresponding vectors and the local truncation error is $\tau_{j}\left(y^{(k+1)}\right)$. Thus,

$$
\max _{j}\left|y_{j}^{(k+1)}-Y_{j}^{(k+1)}\right| \leqslant\left\|A^{-1}\right\| \max _{j}\left|\tau_{j}\left(y^{(k+1)}\right)\right|, k=0,1, \ldots
$$

By a result in [17], we have

$$
\left\|A^{-1}\right\| \leqslant \max _{1 \leqslant i \leqslant N-1}\left\{\left|E_{i}^{(k)}\right|-\left|F_{i}^{(k)}\right|+\left|G_{i}^{(k)}\right|\right\}, k=0,1, \ldots
$$


Theorem 1. Let $Y_{j}^{(k+1)}, j=0, \ldots, N$ be an approximate solution to $y^{(k+1)}(x)$ of equation (3.1) obtained by the proposed method. Then, the following estimate holds

$$
\max _{j}\left|y_{j}^{(k+1)}-Y_{j}^{(k+1)}\right| \leqslant M h^{2}\left\{\exp \left(\frac{-k_{1} x_{j}}{\varepsilon}\right)+\exp \left(\frac{-k_{2}\left(1-x_{j}\right)}{\varepsilon}\right)\right\}
$$

where $k_{1}, k_{2}, M$ are positive constants, independent of $h$ and $\varepsilon$.

\section{Computational results and discussion}

To show the applicability and efficiency of the proposed scheme, it has been implemented to the following problems. Since the exact solution is not known, the maximum pointwise error $E_{N, \varepsilon}^{(k)}$ is calculated by using double mesh principle [8],

$$
E_{N, \varepsilon}^{(k)}=\max _{0 \leqslant x \leqslant 1}\left|\left(y^{(k)}\right)_{i}^{N}-\left(y^{(k)}\right)_{2 i}^{2 N}\right|, \quad k=0,1, \ldots
$$

and computational order $O_{N, \varepsilon}^{(k)}$ is calculated by the following formula

$$
O_{N, \varepsilon}^{(k)}=\log _{2}\left|E_{N, \varepsilon}^{(k)} / E_{2 N, \varepsilon}^{(k)}\right|, \quad k=0,1, \ldots
$$

\begin{tabular}{|c|c|c|c|c|c|c|c|c|c|}
\hline$N$ & 32 & & 64 & & 128 & & 256 & & 512 \\
\hline$\varepsilon$ & $E^{1}$ & $E^{2}$ & $E^{1}$ & $E^{2}$ & $E^{1}$ & $E^{2}$ & $E^{1}$ & $E^{2}$ & $E^{1}$ \\
\hline $2^{-1}$ & $4.84 \mathrm{E}-4$ & $5.50 \mathrm{E}-4$ & $1.31 \mathrm{E}-4$ & $1.49 \mathrm{E}-4$ & $3.39 \mathrm{E}-5$ & $3.88 \mathrm{E}-5$ & $8.66 \mathrm{E}-6$ & $9.91 \mathrm{E}-6$ & $2.18 \mathrm{E}-6$ \\
\hline $2^{-2}$ & $1.54 \mathrm{E}-3$ & $1.78 \mathrm{E}-3$ & $4.28 \mathrm{E}-4$ & $4.98 \mathrm{E}-4$ & $1.13 \mathrm{E}-4$ & $1.32 \mathrm{E}-4$ & $2.90 \mathrm{E}-5$ & $3.42 \mathrm{E}-5$ & $7.37 \mathrm{E}-6$ \\
\hline $2^{-4}$ & $8.86 \mathrm{E}-3$ & $1.08 \mathrm{E}-2$ & $2.82 \mathrm{E}-3$ & $3.52 \mathrm{E}-3$ & $8.17 \mathrm{E}-4$ & $1.04 \mathrm{E}-3$ & $2.21 \mathrm{E}-4$ & $2.86 \mathrm{E}-4$ & $5.78 \mathrm{E}-5$ \\
\hline $2^{-6}$ & $3.87 \mathrm{E}-2$ & $5.05 \mathrm{E}-2$ & $1.23 \mathrm{E}-3$ & $2.16 \mathrm{E}-2$ & $2.91 \mathrm{E}-3$ & $5.58 \mathrm{E}-3$ & $6.93 \mathrm{E}-4$ & $1.35 \mathrm{E}-3$ & $1.68 \mathrm{E}-4$ \\
\hline $2^{-8}$ & $3.68 \mathrm{E}-2$ & $1.30 \mathrm{E}-2$ & $8.95 \mathrm{E}-3$ & $1.52 \mathrm{E}-2$ & $2.12 \mathrm{E}-3$ & $3.49 \mathrm{E}-3$ & $5.22 \mathrm{E}-4$ & $8.62 \mathrm{E}-4$ & $1.30 \mathrm{E}-4$ \\
\hline $2^{-12}$ & $4.05 \mathrm{E}-2$ & $5.39 \mathrm{E}-2$ & 8.33E-3 & $1.35 \mathrm{E}-2$ & $1.87 \mathrm{E}-3$ & $2.91 \mathrm{E}-3$ & $4.46 \mathrm{E}-4$ & $6.84 \mathrm{E}-4$ & $1.11 \mathrm{E}-4$ \\
\hline $2^{-16}$ & $4.84 \mathrm{E}-2$ & $5.23 \mathrm{E}-1$ & $8.33 \mathrm{E}-3$ & $1.35 \mathrm{E}-2$ & $1.87 \mathrm{E}-3$ & $2.91 \mathrm{E}-3$ & $4.45 \mathrm{E}-4$ & $6.82 \mathrm{E}-4$ & $1.09 \mathrm{E}-4$ \\
\hline $2^{-32}$ & $4.84 \mathrm{E}-2$ & $5.23 \mathrm{E}-1$ & $8.33 \mathrm{E}-3$ & $1.35 \mathrm{E}-2$ & $1.87 \mathrm{E}-3$ & $2.91 \mathrm{E}-3$ & $4.45 \mathrm{E}-4$ & $6.82 \mathrm{E}-4$ & $1.09 \mathrm{E}-4$ \\
\hline
\end{tabular}

Table 1. Maximum absolute $\operatorname{error}(E)$ for Example 1 with $\delta=0.5 \varepsilon$

Example 1. Consider the following singularly perturbed nonlinear delay differential equation

$$
\varepsilon y^{\prime \prime}(x)+y(x) y^{\prime}(x-\delta)-y(x)=0
$$

under the interval and boundary conditions

$$
y(0)=1, \quad-\delta \leqslant x \leqslant 0, \quad y(1)=1 .
$$


Table 2. Maximum absolute error and order of convergence for Example 1 with $\varepsilon=2^{-3}$, $\delta=0.5 \varepsilon$

\begin{tabular}{llllll}
\hline$N$ & 32 & 64 & 128 & 256 & 512 \\
\hline$\lambda_{1}=\frac{1}{6}, \quad \lambda_{2}=\frac{1}{3}$ & $8.84 \mathrm{E}-03$ & $2.04 \mathrm{E}-03$ & $4.90 \mathrm{E}-04$ & $1.20 \mathrm{E}-04$ & $2.97 \mathrm{E}-05$ \\
& 2.1155 & 2.0577 & 2.0297 & 2.0145 & 2.0097 \\
$\lambda_{1}=\frac{1}{12}, \quad \lambda_{2}=\frac{5}{12}$ & $1.19 \mathrm{E}-02$ & $2.73 \mathrm{E}-03$ & $6.52 \mathrm{E}-04$ & $1.59 \mathrm{E}-04$ & $3.93 \mathrm{E}-05$ \\
& 2.124 & 2.066 & 2.0358 & 2.0164 & 2.0125 \\
$\lambda_{1}=\frac{1}{18}, \quad \lambda_{2}=\frac{4}{9}$ & $1.30 \mathrm{E}-02$ & $3.00 \mathrm{E}-03$ & $7.14 \mathrm{E}-04$ & $1.74 \mathrm{E}-04$ & $4.31 \mathrm{E}-05$ \\
& 2.1155 & 2.071 & 2.0368 & 2.0133 & 2.0119 \\
$\lambda_{1}=\frac{1}{14}, \lambda_{2}=\frac{3}{7}$ & $1.24 \mathrm{E}-02$ & $2.84 \mathrm{E}-03$ & $6.78 \mathrm{E}-04$ & $1.66 \mathrm{E}-04$ & $4.09 \mathrm{E}-05$ \\
$\lambda_{1}=\frac{1}{30}, \lambda_{2}=\frac{14}{30}$ & 2.1264 & 2.0665 & 2.0301 & 2.0210 & 2.0120 \\
& $1.40 \mathrm{E}-02$ & $3.23 \mathrm{E}-03$ & $7.68 \mathrm{E}-04$ & $1.87 \mathrm{E}-04$ & $4.63 \mathrm{E}-05$ \\
$\lambda_{1}=\frac{1}{24}, \quad \lambda_{2}=\frac{11}{24}$ & 1.1158 & 2.0724 & 2.0381 & 2.0140 & 2.0037 \\
& 2.1253 & 2.0697 & 2.0391 & 2.0159 & 2.0143 \\
\hline
\end{tabular}

Table 1 presents the maximum absolute error for different values of $\lambda_{1}$, $\lambda_{2}, \varepsilon$ and $N$. It can be observed from the results, the error decreases as the mesh size increases. The maximum absolute error and order of convergence are presented in Table 2 for different values of $\lambda_{1}, \lambda_{2}$. It is observed that the proposed method is almost second order convergence.

Figure 1 show the numerical solution for different values of $\delta$. We note that the thickness of the boundary layer increase as the delay increases.

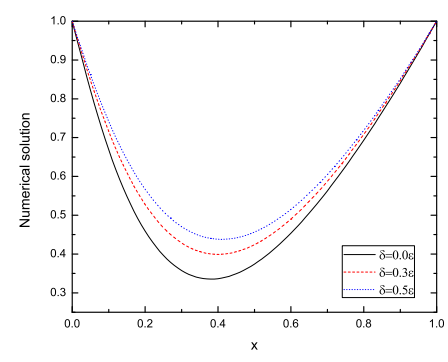

a) $\varepsilon=10^{-1}$

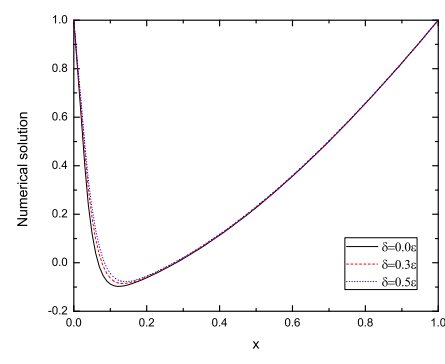

b) $\varepsilon=10^{-2}$

Figure 1. Numerical solution for Example 1.

Example 2. Consider the following singularly perturbed nonlinear delay differential equation

$$
\varepsilon y^{\prime \prime}(x)+2 y^{\prime}(x-\delta)-e^{y(x)}=0
$$

under the interval and boundary conditions

$$
y(0)=0, \quad-\delta \leqslant x \leqslant 0, \quad y(1)=0 .
$$


The maximum absolute error for different values of $\lambda_{1}, \lambda_{2}, \varepsilon$ and $N$ are displayed in Table 3. From the results, it can be observed that the error decreases as the mesh size increases.

Table 3. Maximum absolute error $(E)$ for Example 2 with $\delta=0.5 \varepsilon$

\begin{tabular}{|c|c|c|c|c|c|c|c|c|c|}
\hline$N$ & 32 & & 64 & & 128 & & 256 & & 512 \\
\hline$\varepsilon$ & $E^{1}$ & $E^{2}$ & $E^{1}$ & $E^{2}$ & $E^{1}$ & $E^{2}$ & $E^{1}$ & $E^{2}$ & $E^{1}$ \\
\hline $2^{-1}$ & $7.32 \mathrm{E}-6$ & $9.82 \mathrm{E}-6$ & $1.71 \mathrm{E}-6$ & $2.18 \mathrm{E}-6$ & $4.07 \mathrm{E}-7$ & $5.03 \mathrm{E}-7$ & $9.85 \mathrm{E}-8$ & $1.20 \mathrm{E}-7$ & $2.42 \mathrm{E}-8$ \\
\hline $2^{-2}$ & $2.88 \mathrm{E}-5$ & $3.48 \mathrm{E}-5$ & $6.10 \mathrm{E}-6$ & $7.33 \mathrm{E}-6$ & $1.37 \mathrm{E}-6$ & $1.64 \mathrm{E}-6$ & $3.21 \mathrm{E}-7$ & $3.83 \mathrm{E}-7$ & $7.75 \mathrm{E}-8$ \\
\hline $2^{-3}$ & $4.98 \mathrm{E}-5$ & $5.78 \mathrm{E}-5$ & $9.33 \mathrm{E}-6$ & $1.09 \mathrm{E}-5$ & $1.92 \mathrm{E}-6$ & $2.26 \mathrm{E}-6$ & $4.26 \mathrm{E}-7$ & $5.04 \mathrm{E}-7$ & $9.96 \mathrm{E}-8$ \\
\hline $2^{-4}$ & $5.98 \mathrm{E}-5$ & $6.78 \mathrm{E}-5$ & $9.65 \mathrm{E}-6$ & $1.11 \mathrm{E}-5$ & $1.71 \mathrm{E}-6$ & $1.99 \mathrm{E}-6$ & $3.38 \mathrm{E}-7$ & $3.97 \mathrm{E}-7$ & 7.33E-8 \\
\hline $2^{-8}$ & $2.34 \mathrm{E}-4$ & $2.49 \mathrm{E}-4$ & $4.54 \mathrm{E}-5$ & $4.81 \mathrm{E}-5$ & $6.89 \mathrm{E}-6$ & $7.29 \mathrm{E}-6$ & $9.22 \mathrm{E}-7$ & $9.76 \mathrm{E}-7$ & $1.20 \mathrm{E}-7$ \\
\hline $2^{-12}$ & $2.41 \mathrm{E}-4$ & $2.57 \mathrm{E}-4$ & $5.85 \mathrm{E}-5$ & $6.18 \mathrm{E}-5$ & $1.43 \mathrm{E}-5$ & $1.51 \mathrm{E}-5$ & $3.54 \mathrm{E}-6$ & $3.71 \mathrm{E}-6$ & $8.48 \mathrm{E}-7$ \\
\hline $2^{-16}$ & $2.41 \mathrm{E}-4$ & $2.57 \mathrm{E}-4$ & $5.85 \mathrm{E}-5$ & $6.18 \mathrm{E}-5$ & $1.43 \mathrm{E}-5$ & $1.51 \mathrm{E}-5$ & $3.54 \mathrm{E}-6$ & $3.71 \mathrm{E}-6$ & $8.80 \mathrm{E}-7$ \\
\hline $2^{-18}$ & $2.41 \mathrm{E}-4$ & $2.57 \mathrm{E}-4$ & $5.85 \mathrm{E}-5$ & $6.18 \mathrm{E}-5$ & $1.43 \mathrm{E}-5$ & $1.51 \mathrm{E}-5$ & $3.54 \mathrm{E}-6$ & $3.71 \mathrm{E}-6$ & $8.80 \mathrm{E}-7$ \\
\hline
\end{tabular}

1 Method for $\lambda_{1}=1 / 6, \quad \lambda_{2}=1 / 3$

2 Method for $\lambda_{1}=1 / 12, \quad \lambda_{2}=5 / 12$

Table 4 represents the maximum absolute error and order of convergence for different $\lambda_{1}, \lambda_{2}$ and it is observed that the proposed method is almost second order convergence.

Table 4. Maximum absolute error and order of convergence for Example 2 with $\varepsilon=2^{-3}$, $\delta=0.5 \varepsilon$

\begin{tabular}{llllll}
\hline$N$ & 32 & 64 & 128 & 256 & 512 \\
\hline$\lambda_{1}=\frac{1}{6}, \quad \lambda_{2}=\frac{1}{3}$ & $4.98 \mathrm{E}-05$ & $9.33 \mathrm{E}-06$ & $1.92 \mathrm{E}-06$ & $4.26 \mathrm{E}-07$ & $9.96 \mathrm{E}-08$ \\
& 2.4162 & 2.2808 & 2.1722 & 2.0966 & 2.0176 \\
$\lambda_{1}=\frac{1}{12}, \quad \lambda_{2}=\frac{5}{12}$ & $5.78 \mathrm{E}-05$ & $1.09 \mathrm{E}-05$ & $2.26 \mathrm{E}-06$ & $5.04 \mathrm{E}-07$ & $1.18 \mathrm{E}-07$ \\
& 2.4067 & 2.2699 & 2.1648 & 2.0946 & 2.0163 \\
$\lambda_{1}=\frac{1}{18}, \quad \lambda_{2}=\frac{4}{9}$ & $6.06 \mathrm{E}-05$ & $1.15 \mathrm{E}-05$ & $2.38 \mathrm{E}-06$ & $5.32 \mathrm{E}-07$ & $1.25 \mathrm{E}-07$ \\
& 2.3977 & 2.2726 & 2.1615 & 2.0895 & 2.0189 \\
$\lambda_{1}=\frac{1}{14}, \lambda_{2}=\frac{3}{7}$ & $5.90 \mathrm{E}-05$ & $1.12 \mathrm{E}-05$ & $2.31 \mathrm{E}-06$ & $5.16 \mathrm{E}-07$ & $1.21 \mathrm{E}-07$ \\
$\lambda_{1}=\frac{1}{30}, \lambda_{2}=\frac{14}{30}$ & 2.3972 & 2.2775 & 2.1624 & 2.0924 & 2.0183 \\
& $2.30 \mathrm{E}-05$ & $1.19 \mathrm{E}-05$ & $2.48 \mathrm{E}-06$ & $5.55 \mathrm{E}-07$ & $1.30 \mathrm{E}-07$ \\
$\lambda_{1}=\frac{1}{24}, \lambda_{2}=\frac{11}{24}$ & $6.21 \mathrm{E}-05$ & $1.18 \mathrm{E}-05$ & $2.44 \mathrm{E}-06$ & $5.46 \mathrm{E}-07$ & $1.28 \mathrm{E}-07$ \\
& 2.3958 & 2.2738 & 2.1599 & 2.0928 & 2.0173 \\
\hline
\end{tabular}

The numerical solution for different values of $\delta$ is depicted in Figure 2. It can be observed from the graph that the thickness of the boundary layer increases as the delay increases.

\section{Acknowledgements}

The authors wish to express their gratitude to anonymous referees for very useful comments which improved the presentation of the paper and also the authors would like to thank National Board for Higher Mathematics(NBHM), 


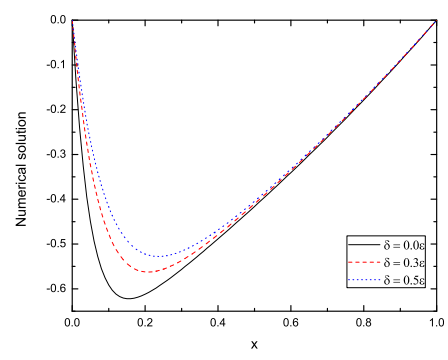

a) $\varepsilon=10^{-1}$

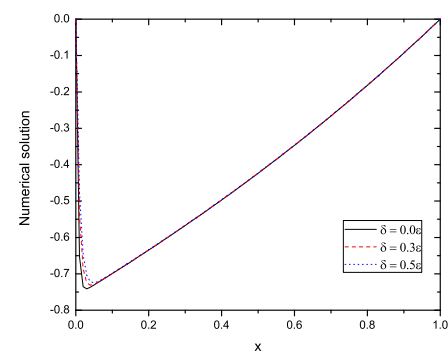

b) $\varepsilon=10^{-2}$

Figure 2. Numerical solution for Example 2.

Government of India for providing financial support under the grant number 2/48(12)/2013/NBHM(R.P.)/R\&D II/1084.

\section{References}

[1] T. Aziz and A. Khan. A spline method for second-order singularly perturbed boundary-value problems. Journal of Computational and Applied Mathematics, 147(2):445-452, 2002. https://doi.org/10.1016/S0377-0427(02)00479-X.

[2] Z. Bartoszewski and A. Baranowska. Solving boundary value problems for second order singularly perturbed delay differential equations by $\varepsilon$-approximate fixed-point method. Mathematical Modelling and Analysis, 20(3):369-381, 2015. https://doi.org/10.3846/13926292.2015.1048759.

[3] R.E. Bellman and R.E. Kalaba. Quasilinearization and nonlinear boundaryvalue problems. Modern analytic and computational methods in science and mathematics. American Elsevier Pub. Co., New York, 1965.

[4] G.F. Carrier. Singular perturbation theory and geophysics. SIAM Review, 12(2):175-193, 1970. https://doi.org/10.1137/1012041.

[5] S. Cengizci. An asymptotic-numerical hybrid method for solving singularly perturbed linear delay differential equations. International Journal of Differential Equations, 2017, 2017. https://doi.org/10.1155/2017/7269450.

[6] P.P. Chakravarthy, S.D. Kumar and R.N. Rao. Numerical solution of second order singularly perturbed delay differential equations via cubic spline in tension. International Journal of Applied and Computational Mathematics, 3(3):17031717, 2017. https://doi.org/10.1007/s40819-016-0204-5.

[7] K.W. Chang and F.A. Howes. Nonlinear singular perturbation phenomena: theory and applications, volume 56. Springer Science \& Business Media, New York, 2012 .

[8] E.P. Doolan, J.J.H. Miller and W.H.A. Schilders. Uniform numerical methods for problems with initial and boundary layers. Advances in numerical computation series. Boole Press Ltd., 1980.

[9] T.C. Hanks. Model relating heat-flow values near, and vertical velocities of mass transport beneath, oceanic rises. Journal of Geophysical Research, 76(2):537544, 1971. https://doi.org/10.1029/JB076i002p00537. 
[10] F.A. Howes. Singular perturbations and differential inequalities, volume 168. American Mathematical Society, Providence, 1976.

[11] M.K. Kadalbajoo and D. Kumar. A computational method for singularly perturbed nonlinear differential-difference equations with small shift. Applied Mathematical Modelling, 34(9):2584-2596, 2010. https://doi.org/10.1016/j.apm.2009.11.021.

[12] M.K. Kadalbajoo and K.K. Sharma. Numerical treatment for singularly perturbed nonlinear differential difference equations with negative shift. Nonlinear Analysis: Theory, Methods \& Applications, 63(5):e1909-e1924, 2005. https://doi.org/10.1016/j.na.2005.02.098.

[13] C.G. Lange and R.M. Miura. Singular perturbation analysis of boundary value problems for differential-difference equations. IV. A nonlinear example with layer behavior. Studies in Applied Mathematics, 84(3):231-273, 1991. https://doi.org/10.1002/sapm1991843231.

[14] R. Narasimhan. Singularly perturbed delay differential equations and numerical methods. In V. Sigamani, J.J.H. Miller, R. Narasimhan, P. Mathiazhagan and F. Victor(Eds.), Differential Equations and Numerical Analysis: Tiruchirappalli, India, January 2015, pp. 41-62. Springer India, New Delhi, 2016. https://doi.org/10.1007/978-81-322-3598-9_3.

[15] R.E. O'Malley. Introduction to singular perturbations. North-Holland Series in Applied Mathematics \& Mechanics. Academic Press Inc., New York, 1974.

[16] R.N. Rao and P.P. Chakravarthy. A numerical patching technique for singularly perturbed nonlinear differential-difference equations with a negative shift. Applied Mathematics, 2(2):11-20, 2012. https://doi.org/10.5923/j.am.20120202.04.

[17] J.M. Varah. A lower bound for the smallest singular value of a matrix. Linear Algebra and its Applications, 11(1):3-5, 1975. https://doi.org/10.1016/00243795(75)90112-3.

[18] R. Vulanovic, P.A. Farrell and P. Lin. Numerical solution of nonlinear singular perturbation problems modeling chemical reactions. In Applications of Advanced Computational Methods for Boundary and Interior Layers, pp. 192-213. Press, 1993. 